\title{
Patients with seronegative RA have more inflammatory activity compared with patients with seropositive RA in an inception cohort of DMARD-naïve patients classified according to the 2010 ACR/EULAR criteria
}

\author{
Lena Bugge Nordberg, ${ }^{1}$ Siri Lillegraven, ${ }^{1}$ Elisabeth Lie, ${ }^{1}$ Anna-Birgitte Aga, ${ }^{1}$ \\ Inge Christoffer Olsen, ${ }^{1}$ Hilde Berner Hammer, ${ }^{1}$ Till Uhlig, ${ }^{1}$ Maria Karolina Jonsson, ${ }^{1,2}$ \\ Désirée van der Heijde, ${ }^{1,3}$ Tore K Kvien, ${ }^{1}$ Espen Andre Haavardsholm, ${ }^{1}$ and the \\ ARCTIC working group
}

\begin{abstract}
Handling editor Gerd R Burmester

${ }^{1}$ Department of Rheumatology, Diakonhjemmet Hospital, Oslo, Norway

${ }^{2}$ Department of Rheumatology, Haukeland University Hospital, Bergen, Norway ${ }^{3}$ Leiden University Medical Center, Leiden, Netherlands
\end{abstract}

\section{Correspondence to} Dr Lena Bugge Nordberg, Department of Rheumatology, Diakonhjemmet Hospital, Box 23 Vinderen,

Oslo N-0319, Norway; lenabuggenordberg@gmail. com

Received 6 November 2015 Revised 16 March 2016 Accepted 29 March 2016 Published Online First 19 April 2016
CrossMark

\section{SLinked}

http://dx.doi.org/10.1136/ annrheumdis-2016-209629

To cite: Nordberg $L B$,

Lillegraven $\mathrm{S}$, Lie $\mathrm{E}$, et al.

Ann Rheum Dis

2017:76:341-345.
ABSTRACT

Objectives To compare the presentation of seropositive and seronegative early rheumatoid arthritis (RA) in disease-modifying antirheumatic drug (DMARD)naive patients classified according to the 2010 American College of Rheumatology (ACR)/European League

Against Rheumatism (EULAR) criteria.

Methods All patients had symptom duration from first swollen joint $<2$ years and were DMARD naive with an indication for DMARD treatment. Patients were stratified as seropositive (positive rheumatoid factor (RF)+ and/or anticitrullinated peptide antibody (ACPA)+) or seronegative (RF- and ACPA-), and disease characteristics were compared between groups.

Results A total of 234 patients were included, and 36 (15.4\%) were seronegative. Ultrasonography (US) scores for joints (median 55 vs 25, p<0.001) and tendons (median 3 vs $0, p<0.001$ ), number of swollen joints (median 17 vs $8, p<0.001$ ), disease activity score (DAS; mean 3.9 vs $3.4, p=0.03$ ) and physician global assessment (mean 49.1 vs $38.9, p=0.006$ ) were significantly higher in seronegative patients compared with seropositive. Total van der Heijde-modified Sharp score, Richie Articular Index and patient-reported outcome measures were similar between groups. Conclusions Seronegative patients had higher levels of inflammation, assessed both clinically and by US, than seropositive patients. These differences may reflect the high number of involved joints required for seronegative patients to fulfil the 2010 ACR/EULAR classification criteria for RA.

Trial registration number NCT01205854; Pre-results.

\section{INTRODUCTION}

The identification of rheumatoid factor (RF) and anticitrullinated protein antibodies (ACPAs) has led to the recognition of the subgroups of seropositive and seronegative rheumatoid arthritis (RA). ${ }^{1}$ Serological status has become an important factor in diagnosis and prognostication of the disease. Patients with seropositive RA share certain genetic and environmental risk factors and have been shown to have a more severe disease course. ${ }^{2} 3$ Less is known about seronegative RA, but evidence of genetic associations for ACPA-negative RA has been found. ${ }^{4}$ The clinical presentation and disease course of seronegative RA are usually reported as less severe than for seropositive RA, although studies are conflicting. ${ }^{5}$

The 2010 American College of Rheumatology (ACR)/European League Against Rheumatism (EULAR) classification criteria for RA have led to the redefinition of the patient population by increased weighting of serology. ${ }^{6}$ Compared with the 1987 criteria, $^{7}$ the new criteria appear to result in an increased prevalence of classifiable RA with a milder disease course. ${ }^{8}$ In previous studies, the new criteria do not seem to alter the ratio of patients with seronegative RA to patients with seropositive $\mathrm{RA},{ }^{9}{ }^{10}$ but it is unknown how they affect the presentation of the two subgroups.

Our aim was to compare the clinical presentation of seronegative and seropositive RA in diseasemodifying antirheumatic drug (DMARD)-naïve patients classified according to the 2010 criteria, with a focus on inflammatory activity assessed clinically and by ultrasonography (US).

\section{METHODS}

\section{Patients}

Patients with RA who fulfilled the 2010 ACR/ EULAR classification criteria were recruited at 11 Norwegian rheumatology centres between 2010 and 2013 and included in the Aiming for Remission in Rheumatoid Arthritis: a Randomized Trial Examining the Benefit of Ultrasonography in a Clinical Tight Control Regiment (ARCTIC) trial (ClinicalTrials.gov identifier NCT01205854). All patients provided written informed consent. All patients had symptom duration of less than 2 years and were DMARD naïve with an indication for DMARD treatment. Patients were stratified as seropositive (RF (IgM or IgA)+, ACPA+ or both) or seronegative (both RF- and ACPA-).

\section{Data collection}

The data collection included demographic data, ACPA, RF, erythrocyte sedimentation rate (ESR, 
$\mathrm{mm} / \mathrm{h}$ ), C reactive protein (CRP, mg/L), Ritchie Articular Index, 44 swollen joint count (SJC) and the patient's and physician's assessment of disease activity on $0-100 \mathrm{~mm}$ Visual Analogue Scales (VASs). The original disease activity score (DAS) was calculated. $^{11}$

RF and ACPA were analysed centrally by ELISA and fluorescence enzyme immunoassay, respectively. A positive result was defined as any value $\geq 10 \mathrm{IU} / \mathrm{mL}$ for ACPA and $25 \mathrm{IU} / \mathrm{mL}$ for RF. A low antibody level was defined as any value $>$ the defined upper limit of normal (ULN) and $\leq 3 \times$ ULN, and a high antibody level as a value $>3 \times$ ULN. $^{6}$

The impact of disease was assessed by the Rheumatoid Arthritis Impact of Disease (RAID) score, Patient-Reported Outcome Measurement Information System (PROMIS), 36-item Short Form Health Survey (SF-36), EuroQol-5 Dimensions (EQ-5D), fatigue VAS and pain VAS.

US examinations were performed by experienced ultrasonographers using a validated $0-3$ semiquantitative scoring system for grey scale (GS) and power Doppler (PD). ${ }^{12}$ The 36 joints were as follows: metacarpophalangeal (MCP) 1-5, proximal interphalangeal (PIP) 2-3, radiocarpal (RCJ), intercarpal (ICJ), distal radioulnar (DRUJ), elbow, knee, talocrural and metatarsophalangeal (MTP) 1-5. Tenosynovitis of the tibialis posterior tendon and the extensor carpi ulnaris tendon was also evaluated. An atlas was available as reference during scorings. For dichotomisation of ultrasonographic synovitis, we used a definition of GS score $\geq 2$ and/or a PD score $\geq 1$ based on previous publications. ${ }^{13}$ For the wrist, ultrasonographic synovitis was defined as present if RCJ, DRUJ or ICJ had ultrasonographic synovitis.

Radiographs of hands and feet were acquired and scored according to the van der Heijde-modified Sharp score (vdHSS). ${ }^{14}$ Two experienced readers blinded for patient identity and clinical information scored all radiographs.

\section{Statistical analyses}

Statistical analyses were performed using Stata 14. Continuous variables are presented as means (SD) or medians $(25$ th, 75 th centiles) according to distribution, and dichotomous variables are presented as frequencies and percentages. Disease characteristics were compared between subgroups using independent samples $t$ test, Mann-Whitney $U$ test or $\chi^{2}$ test as appropriate. Statistical tests were two-sided and $\mathrm{p}$ values less than 0.05 were considered statistically significant. For all variables, less than 5\% of data were missing, thus no imputation of data was done.

\section{RESULTS}

A total of 234 patients with mean age (SD) 51.5 (13.7) years and median disease duration $(25,75$ centiles $) 5.6(2.8,10.2)$ months were included. Overall, 145 patients $(62.0 \%)$ were women, and 36 patients (15.4\%) were seronegative.

Seronegative patients were older than seropositive patients (55.8 years vs 50.7 years, $\mathrm{p}=0.04$ ), while the gender distribution was similar. Similar percentages of patients fulfilled the 1987 ACR criteria (61.1\% seronegative vs $68.7 \%$ seropositive patients, $p=0.37$ ). In patients who did not fulfil the 1987 criteria, few statistically significant differences between seronegative and seropositive patients were found with regard to which of the 1987 criteria items were fulfilled. Seronegative patients more often had arthritis of three or more areas $(71 \%$ vs $26 \%, p=0.001)$ and arthritis of hand joints $(79 \%$ vs $48 \%$, $\mathrm{p}=0.04)$.

US scores, SJC, DAS and physician global were significantly higher in seronegative compared with seropositive patients

\begin{tabular}{|c|c|c|c|}
\hline Variable & $\begin{array}{l}\text { Seronegative } \\
\text { RA } \\
(n=36)\end{array}$ & $\begin{array}{l}\text { Seropositive } \\
\text { RA } \\
(n=198)\end{array}$ & $\mathrm{p}$ Value \\
\hline Age, years & $55.8(15.4)$ & $50.7(13.3)$ & 0.04 \\
\hline Female, $\mathrm{n}(\%)$ & $21(58.3)$ & $124(62.6)$ & 0.63 \\
\hline Disease duration, months & $4.8[2.5-11.7]$ & 5.9 [2.9-9.9] & 0.89 \\
\hline Positive for ACPA, n (\%) & NA & $187(94.4)$ & \\
\hline Positive for RF IgM & NA & $154(77.8)$ & \\
\hline Positive for RF IgA & NA & $110(55.6)$ & \\
\hline DAS* $^{*}$ & $3.9(1.2)$ & $3.4(1.2)$ & 0.03 \\
\hline $44 \mathrm{SJC}$ & 17 [11-25] & 8 [4-13] & $<0.001$ \\
\hline CRP, mg/L & $7.3[3.0-27.0]$ & $7.0[3.0-18.0]$ & 0.48 \\
\hline $\mathrm{ESR}, \mathrm{mm} / \mathrm{h}$ & $14.0[10.0-29.0]$ & $20.5[12.0-33.0]$ & 0.21 \\
\hline Ritchie Articular Index & $7.5[3.5-14.0]$ & $7.0[4.0-13.0]$ & 0.73 \\
\hline Physician global VAS 0-100 mm & $49.1(21.1)$ & $38.9(20.2)$ & 0.006 \\
\hline Patient global VAS 0-100 mm & $50.0(26.6)$ & $49.6(23.9)$ & 0.92 \\
\hline Total US joint scoret & $55[31-82]$ & $25[15-40]$ & $<0.001$ \\
\hline US PD joint score & $16[7-27]$ & $7[3-14]$ & $<0.001$ \\
\hline US GS joint score & $37[23-58]$ & $18.5[10-27]$ & $<0.001$ \\
\hline Joints with US synovitis & 16 [9-20] & $6[3-10]$ & $<0.001$ \\
\hline Total US tendon score & $3[0-7]$ & $0[0-3]$ & $<0.001$ \\
\hline US PD tendon score & $1[0-4]$ & $0[0-2]$ & 0.008 \\
\hline US GS tendon score & $2[0-3]$ & $0[0-2]$ & $<0.001$ \\
\hline $\begin{array}{l}\text { Total van der Heijde-modified } \\
\text { Sharp score }\end{array}$ & $5.5[2.0-13.0]$ & $4.0[1.5-8.0]$ & 0.20 \\
\hline Joint space narrowing & $1.5[0.0-6.0]$ & $1.0[0.0-3.0]$ & 0.06 \\
\hline Erosion score & $3.0[1.0-6.5]$ & $3.0[1.0-4.5]$ & 0.43 \\
\hline EQ-5D & $0.66[0.11-0.76]$ & $0.66[0.23-0.73]$ & 0.84 \\
\hline Pain VAS 0-100 mm & $45.5(26.3)$ & $48.1(23.5)$ & 0.55 \\
\hline Fatigue VAS 0-100 mm & $41.5(29.8)$ & $40.0(28.4)$ & 0.78 \\
\hline RAID score & $4.7(2.3)$ & $4.4(2.1)$ & 0.53 \\
\hline PROMIS HAQ & 36.3 [13.8-58.8] & $29.5[17.5-48.8]$ & 0.78 \\
\hline SF-36 PCS & $32.5(7.9)$ & $36.6(9.7)$ & 0.37 \\
\hline SF-36 MCS & $48.6(11.9)$ & $49.3(10.3)$ & 0.75 \\
\hline
\end{tabular}

Bold indicates p-values less than 0.05 .

Values are median [25th-75th centiles] or mean (SD), unless otherwise indicated

*Original disease activity score based on ESR, patient global, 44 SJC and Ritchie Articular Index.

†Ultrasonography (US) semiquantitative scoring system with ranges $0-3$ for grey scale (GS) and power Doppler (PD) in 36 joints and 4 tendons. (Total US joint score, range: $0-216$. Total US tendon score, range: $0-24$.)

ACPA, anticitrullinated peptide antibody; CRP, $C$ reactive protein; DAS, disease activity score; EQ-5D, EuroQol-5 Dimensions (UK weighted); ESR, erythrocyte sedimentation rate; MCS, Mental Components Summary; PCS, Physical Components Summary; PROMIS HAQ, Patient-Reported Outcome Measurement Information System Health Assessment Questionnaire; RA, rheumatoid arthritis; RAID, Rheumatoid Arthritis Impact of Disease (range from 0 to 10, with higher scores indicating greater impact of disease); RF, rheumatoid factor; SF-36, 36-item Short Form Health Survey; SJC, swollen joint count; VAS, Visual Analogue Scale.

(table 1). Limiting analyses to patients meeting the 1987 criteria for RA revealed similar results; however, the differences were numerically smaller (data not shown) and interpretation is difficult as patients fulfilling the 1987 criteria only were not included.

SJC was significantly higher in seronegative patients compared with patients with high levels of RF/ACPA $(p<0.001)$, but did not differ substantially compared with patients with low antibody levels $(p=0.18)$. There was a statistically significant difference between patients with high and low antibody levels for SJC $(p=0.02)$ (figure 1A). Seronegative patients differed compared with patients with both low and high 

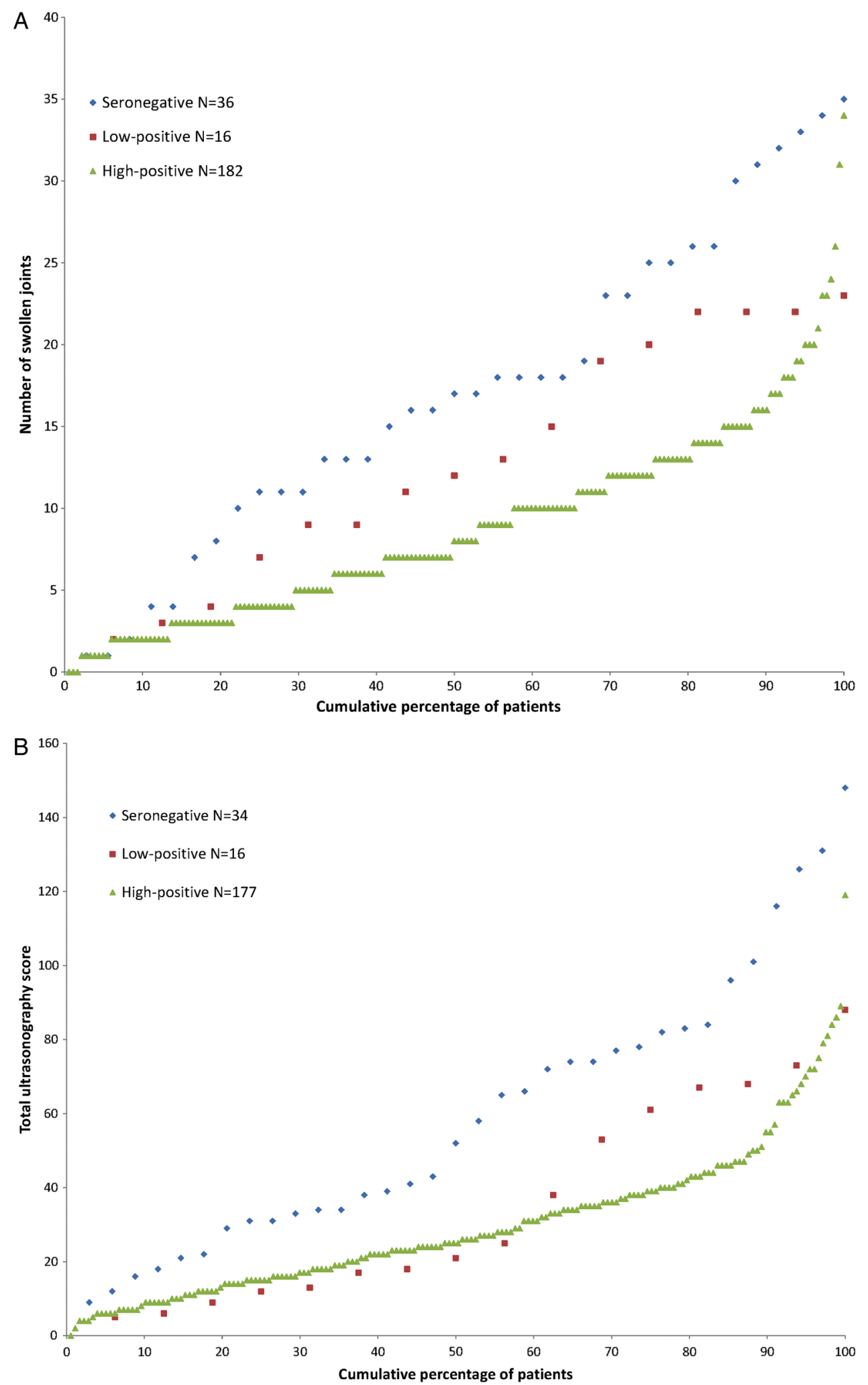

Figure 1 Cumulative probability plots of (A) 44 swollen joint count (SIC) and (B) total ultrasonography (US) score. Groups according to antibody levels: seronegative $(<\mathrm{ULN})$, low positive (ULN to $\leq 3 \times \mathrm{ULN})$ and high positive $(>3 \times \mathrm{ULN})$. (A) 44 SJC. (B) Total US score. ULN, upper limit of normal.

antibody levels with regard to US scores $(p=0.017$ and $<0.001$, respectively), whereas no significant differences were found between patients with low and high antibody levels $(\mathrm{p}=0.73$, figure $1 \mathrm{~B})$.
Joint tenderness, patient-reported outcome measures and radiography scores were similar between groups (table 1). In seronegative patients, the MCP joints were more often swollen and positive for ultrasonographic synovitis than in seropositive 


\begin{tabular}{|c|c|c|c|c|c|c|c|}
\hline \multicolumn{4}{|c|}{ Clinically swollen joints } & \multicolumn{4}{|c|}{ Joints with ultrasonographic synovitis } \\
\hline \multicolumn{2}{|c|}{$\begin{array}{l}\text { Seronegative patients } \\
\mathrm{N}=36 \text { patients, } 72 \text { joints }\end{array}$} & \multicolumn{2}{|c|}{$\begin{array}{l}\text { Seropositive patients } \\
\mathrm{N}=198 \text { patients, } 396 \text { joints }\end{array}$} & \multicolumn{2}{|c|}{$\begin{array}{l}\text { Seronegative patients } \\
\mathrm{N}=34 \text { patients, } 68 \text { joints }\end{array}$} & \multicolumn{2}{|c|}{$\begin{array}{l}\text { Seropositive patients } \\
\mathrm{N}=194 \text { patients, } 388 \text { joints }\end{array}$} \\
\hline Joint & $\begin{array}{l}\text { Percentage affected } \\
\text { (ordered according to } \\
\text { frequency) (\%) }\end{array}$ & Joint & $\begin{array}{l}\text { Percentage affected } \\
\text { (ordered according to } \\
\text { frequency) (\%) }\end{array}$ & Joint & $\begin{array}{l}\text { Percentage affected } \\
\text { (ordered according to } \\
\text { frequency) (\%) }\end{array}$ & Joint & $\begin{array}{l}\text { Percentage affected } \\
\text { (ordered according to } \\
\text { frequency) (\%) }\end{array}$ \\
\hline Wrist & 75.0 & MTP3 & 43.2 & Wrist & 82.4 & MTP3 & 38.1 \\
\hline MCP3 & 66.7 & MTP2 & 41.7 & MCP2 & 73.5 & Wrist & 37.9 \\
\hline MCP2 & 65.3 & PIP2 & 40.9 & MCP3 & 63.2 & MTP2 & 37.9 \\
\hline PIP3 & 61.1 & MCP2 & 40.4 & MCP1 & 55.9 & MCP2 & 33.3 \\
\hline MCP1 & 59.7 & PIP3 & 38.1 & MCP4 & 48.5 & MTP1 & 30.8 \\
\hline PIP2 & 56.9 & Wrist & 32.8 & MTP2 & 45.6 & MCP1 & 26.8 \\
\hline MTP3 & 54.2 & MCP3 & 30.3 & PIP2 & 45.6 & MTP4 & 26.5 \\
\hline PIP4 & 51.4 & MCP1 & 29.8 & PIP3 & 44.1 & MCP3 & 22.9 \\
\hline MTP2 & 51.4 & MTP4 & 28.3 & MCP5 & 44.1 & PIP2 & 22.7 \\
\hline MCP5 & 48.6 & PIP4 & 20.5 & MTP1 & 41.2 & MTP5 & 21.9 \\
\hline
\end{tabular}

The 10 most frequently affected joints are presented. Joints were assessed bilaterally and analysed on an aggregated level.

MCP, meatacarpophalangeal; MTP, metatarsophalangeal; PIP, proximal interphalangeal.

patients, while seropositive patients had relatively more frequent involvement of MTP joints (table 2).

\section{DISCUSSION}

We found patients with seronegative RA to have higher disease activity, assessed both clinically and by US, than seropositive patients, despite similar disease duration. This is in contrast with most previous studies, which have shown either no difference between the subgroups or more severe disease in seropositive patients. ${ }^{15-18}$

The 2010 criteria put great emphasis on RF and ACPA status. While seropositive patients can fulfil the criteria with only one affected joint, more than 10 involved joints are required for seronegative patients to fulfil the criteria. ${ }^{6}$ Consequently, it was not unexpected that seronegative patients had more joint involvement compared with seropositive patients. However, we find the differences to be larger than expected. Seronegative patients had a median of 17 swollen joints (the criteria require 11 swollen and/or tender joints to get a maximum score), compared with a median number of swollen joints of 8 in the seropositive group. In our cohort, $38.9 \%$ of the seronegative patients did not fulfil the 1987 criteria, indicating that the new criteria do capture seronegative patients excluded by the old criteria. In line with our findings, more severe disease was seen in seronegative than in seropositive patients with early arthritis in the Canadian Early Arthritis Cohort (CATCH). ${ }^{19}$ The majority of these patients had already received DMARD treatment, hampering the interpretation of the results.

It has been suggested that seropositive patients are referred to a rheumatologist regardless of disease severity, while seronegative patients with mild disease are less frequently referred than seronegative patients with severe disease. ${ }^{19}$ Similarly, the rheumatologist may more often apply the RA criteria in seronegative patients with severe disease, while a diagnosis of RA is considered regardless of disease severity in seropositive patients. Such mechanisms may have affected recruitment of patients to the current study and contributed to the relatively low proportion of seronegative patients (15.4\%). Higher numbers of seronegative patients in a previous Norwegian RA cohort based on 1987 ACR criteria $(34.5 \%$ seronegative) indicate that the introduction of the 2010 criteria changed the ratio of patients with seronegative
RA to patients with seropositive RA, although we cannot know for sure how different mechanisms contributed to this. ${ }^{20}$

As the seronegative patients had markedly higher disease activity, it is surprising that assessments of pain and quality of life were similar between the groups. Although seronegative patients had more inflammatory activity, radiographic joint damage was not statistically significantly different between groups. These findings may reflect different pathophysiological mechanisms in the subgroups.

A limitation of this study is that only patients classified as having RA according to the 2010 ACR/EULAR criteria were included, thus it was not possible to assess patients only fulfilling the 1987 criteria for RA. Strengths of our study are the extensive data collection aiming to include all newly diagnosed patients with RA at the participating centres, with radiographic and ultrasonographic examinations of all patients. Questions regarding the performance of the 2010 ACR/EULAR criteria in seronegative patients have previously been partly addressed, but with limiting supporting data. ${ }^{21}$ To our knowledge, this is the first study to specifically examine the presentation of DMARD-naïve seronegative RA in patients classified according to the new criteria.

In summary, we found seronegative patients to have higher disease activity, assessed both clinically and by US, than seropositive patients. This finding may reflect a limitation in the performance of the 2010 ACR/EULAR classification criteria. Our results suggest that the new criteria capture a different subset of patients with seronegative RA, with more inflammatory activity, compared with the 1987 criteria.

Acknowledgements The authors wish to thank the patients for participating in this study, the local rheumatology staff for data collection and research coordinators Ellen Moholt and Camilla Fongen for their contributions.

Collaborators The ARCTIC working group: Hallvard Fremstad, Tor Magne Madland, Åse Stavland Lexberg, Hilde Haukeland, Erik Rødevand, Christian Høili, Hilde Stray, Anne Noraas Bendvold, Dag Magnar Soldal, Gunnstein Bakland.

Contributors All authors were involved in drafting the article or revising it critically for important intellectual content and approved the final manuscript to be submitted and agreed to be accountable for all aspects of the work. Conception and design of the study: EAH, SL, LBN, A-BA, EL, ICO, HBH, TU, DvdH and TKK. Acquisition of data: $E A H, A-B A, H B H, T U, J M K$ and the ARCTIC study group. Analysis and interpretation of data: LBN, ICO, EAH, SL, EL, A-BA, DvdH and TKK. 
Funding The study has received grants from the Norwegian Research Council, the South-East Health Region in Norway, The Norwegian Rheumatism Association, the Norwegian Women's Public Health Association and unrestricted grant support from AbbVie, Pfizer, MSD, Roche and UCB.

Competing interests $\mathrm{HBH}$ has received speakers's fees from BMS, UCB, Roche, Abbvie and Pfizer, $v$ TKK has received grants from Abbvie, BMS, MSD/ Schering-Plough, Pfizer/Wyeth, Roche, UCB and payment for lectures from Abbvie, Astra Zeneka, MSD/Schering-Plough, Pfizer/Wyeth, Roche, UCB, Celltrion and Eli Lily. EAH has received personal fees for consultancy, payment for lectures or development of educational material from UCB Pharma, Roche, Abbvie and Pfizer.

Ethics approval Local regional ethics committee.

Provenance and peer review Not commissioned; externally peer reviewed.

\section{REFERENCES}

1 Klareskog L, Catrina Al, Paget S. Rheumatoid arthritis. Lancet 2009; 373:659-72.

2 Syversen SW, Goll GL, van der Heijde D, et al. Prediction of radiographic progression in rheumatoid arthritis and the role of antibodies against mutated citrullinated vimentin: results from a 10-year prospective study. Ann Rheum Dis 2010;69:345-51.

3 Han B, Diogo D, Eyre $S$, et al. Fine mapping seronegative and seropositive rheumatoid arthritis to shared and distinct HLA alleles by adjusting for the effects of heterogeneity. Am J Hum Genet 2014;94:522-32.

4 Daha NA, Toes RE. Rheumatoid arthritis: are ACPA-positive and ACPA-negative RA the same disease? Nat Rev Rheumatol 2011;7:202-3.

5 Ajeganova S, Huizinga TW. Rheumatoid arthritis: seronegative and seropositive RA: alike but different? Nat Rev Rheumatol 2015;11:8-9.

6 Aletaha D, Neogi T, Silman AJ, et al. 2010 rheumatoid arthritis classification criteria: an American College of Rheumatology/European League Against Rheumatism collaborative initiative. Ann Rheum Dis 2010;69:1580-8.

7 Arnett FC, Edworthy SM, Bloch DA, et al. The American Rheumatism Association 1987 revised criteria for the classification of rheumatoid arthritis. Arthritis Rheum 1988:31:315-24.

8 Radner H, Neogi T, Smolen JS, et al. Performance of the 2010 ACR/EULAR classification criteria for rheumatoid arthritis: a systematic literature review. Ann Rheum Dis 2014;73:114-23.
9 Cader MZ, Filer A, Hazlehurst J, et al. Performance of the 2010 ACR/EULAR criteria for rheumatoid arthritis: comparison with 1987 ACR criteria in a very early synovitis cohort. Ann Rheum Dis 2011;70:949-55.

10 de Hair MJ, Lehmann KA, van de Sande MG, et al. The clinical picture of rheumatoid arthritis according to the 2010 American College of Rheumatology/ European League Against Rheumatism criteria: is this still the same disease? Arthritis Rheum 2012;64:389-93.

11 van der Heijde DM, van 't Hof M, van Riel PL, et al. Development of a disease activity score based on judgment in clinical practice by rheumatologists. J Rheumatol 1993;20:579-81.

12 Hammer HB, Bolton-King P, Bakkeheim V, et al. Examination of intra and interrater reliability with a new ultrasonographic reference atlas for scoring of synovitis in patients with rheumatoid arthritis. Ann Rheum Dis 2011;70:1995-8.

13 van Onna M, Ten Cate DF, Tsoi KL, et al. Assessment of disease activity in patients with rheumatoid arthritis using optical spectral transmission measurements, a non-invasive imaging technique. Ann Rheum Dis 2016;75: 511-18.

14 van der Heijde D. How to read radiographs according to the Sharp/van der Heijde method. J Rheumatol 2000;27:261-3.

15 Farragher TM, Lunt M, Plant D, et al. Benefit of early treatment in inflammatory polyarthritis patients with anti-cyclic citrullinated peptide antibodies versus those without antibodies. Arthritis Care Res (Hoboken) 2010;62:664-75.

16 Forslind $K$, Ahlmén M, Eberhardt $K$, et al. Prediction of radiological outcome in early rheumatoid arthritis in clinical practice: role of antibodies to citrullinated peptides (anti-CCP). Ann Rheum Dis 2004;63:1090-5.

17 Kastbom A, Strandberg G, Lindroos A, et al. Anti-CCP antibody test predicts the disease course during 3 years in early rheumatoid arthritis (the Swedish TIRA project). Ann Rheum Dis 2004;63:1085-9.

18 Ursum J, Bos WH, van Dillen $\mathrm{N}$, et al. Levels of anti-citrullinated protein antibodies and IgM rheumatoid factor are not associated with outcome in early arthritis patients: a cohort study. Arthritis Res Ther 2010;12:R8.

19 Barra L, Pope JE, Orav JE, et al. Prognosis of seronegative patients in a large prospective cohort of patients with early inflammatory arthritis. J Rheumatol 2014:41:2361-9.

20 Haavardsholm EA, Bøyesen P, Østergaard M, et al. Magnetic resonance imaging findings in 84 patients with early rheumatoid arthritis: bone marrow oedema predicts erosive progression. Ann Rheum Dis 2008;67:794-800.

21 Mjaavatten MD, Bykerk VP. Early rheumatoid arthritis: the performance of the 2010 ACR/EULAR criteria for diagnosing RA. Best Pract Res Clin Rheumatol 2013;27:451-66. 